\title{
Finite difference approximation eigenvibrations of a bar with oscillator
}

\author{
D. M. Korosteleva ${ }^{1}$, L. N. Koronova ${ }^{2}$, K. O. Levinskaya ${ }^{2}$, and S. I. Solov'eve, \\ ${ }^{1}$ Kazan State Power Engineering University, 51 Krasnoselskaya Street, Kazan, 420066, Russian \\ Federation \\ ${ }^{2}$ Kazan Federal University, 18 Kremlevskaya Street, Kazan, 420008, Russian Federation
}

\begin{abstract}
The second-order ordinary differential spectral problem governing eigenvibrations of a bar with attached harmonic oscillator is investigated. We study existence and properties of eigensolutions of formulated bar-oscillator spectral problem. The original second-order ordinary differential spectral problem is approximated by the finite difference mesh scheme. Theoretical error estimates for approximate eigenvalues and eigenfunctions of this mesh scheme are established. Obtained theoretical results are illustrated by computations for a model problem with constant coefficients. Theoretical and experimental results of this paper can be developed and generalized for the problems on eigenvibrations of complex mechanical constructions with systems of harmonic oscillators.
\end{abstract}

\section{Introduction}

We investigate the longitudinal eigenvibrations of a bar of length $l$. Denote by $\rho(x)$, $E(x)$ and $S(x)$ the density, the elasticity modulus of the bar material and the square of transversal cut of the bar at the point $x \in \bar{\Omega}=[0, l], \Omega=(0, l)$. Suppose that the end $x=0$ is fixed, a spring of stiffness $K$ is attached to the end $x=l$ and a load of mass $M$ is attached to the spring. By $w(x, t)$ we denote the deflection of the bar cross-section with coordinate $x \in \bar{\Omega}$ at time $t>0$ and by $\eta(t)$ the longitudinal deflection of the load of mass $M$ from the equilibrium position at time $t>0$. These functions satisfy the following system of equations

$$
\frac{\partial}{\partial x}\left(p(x) \frac{\partial w(x, t)}{\partial x}\right)-r(x) \frac{\partial^{2} w(x, t)}{\partial t^{2}}=0, x \in \Omega, t>0,
$$

\footnotetext{
*Corresponding author: sergey.solovev.kpfu@mail.ru
} 


$$
\begin{gathered}
w(0, t)=p(l) \frac{\partial w(l, t)}{\partial x}-K \eta(t)-K w(l, t)=0, t>0, \\
M \eta^{\prime \prime}(t)=-K \eta(t)+K w(l, t), t>0
\end{gathered}
$$

with coefficients $p(x)=S(x) E(x), r(x)=S(x) \rho(x), x \in \bar{\Omega}, t>0$.

The eigenvibrations of the mechanical bar-oscillator system are described by the functions $w(x, t)=u(x) \sin (\omega t), x \in \bar{\Omega}, \eta(t)=z \sin (\omega t), t>0$, with constants $z$ and $\omega$. Let us denote $\sigma=K / M, \lambda=\omega^{2}$, then from (3) it follows that

$$
\sigma z-\lambda z=u(l) \sigma
$$

For $\lambda \neq \sigma$, by (4) we have the formula $z=\sigma u(l) /(\sigma-\lambda)$, and using (1)-(3) we formulate the following differential spectral problem with rational dependence on the spectral parameter: find $\lambda$ and $u(x), x \in \bar{\Omega}$, satisfying the following equations

$$
\begin{gathered}
\left(p(x) u^{\prime}(x)\right)^{\prime}+\lambda r(x) u(x)=0, x \in \Omega, \\
u(0)=p(l) u^{\prime}(l)-\lambda(\sigma-\lambda)^{-1} K u(l)=0 .
\end{gathered}
$$

For $\lambda=\sigma$, by (6) we derive the equality $u(l)=0$, and then using (1) and (2), we get the following limit linear spectral problem: find $\lambda^{(0)}=\sigma$ and $u(x), x \in \bar{\Omega}$, satisfying the following equations

$$
\begin{gathered}
\left(p(x) u^{\prime}(x)\right)^{\prime}-\lambda^{(0)} r(x) u(x)=0, x \in \Omega, \\
u(0)=u(l)=0 .
\end{gathered}
$$

In the present paper, the rational bar-oscillator differential spectral problem (5), (6), is approximated by the finite difference scheme. The theoretical error estimates of approximate eigensolutions of this finite difference scheme are derived. Obtained theoretical results are illustrated by computations for a problem with constant coefficients.

Spectral approximations for compact operators are investigated in the papers [1-4]. Generalizations of spectral approximations for holomorphic Fredholm operator functions are derived in the papers $[5,6]$. Preconditioned iterative methods for solving linear spectral problems are proposed and investigated in the papers [7-14]. Iterative methods for solving spectral problems with nonlinear parameter are proposed and investigated in the papers [15-26]. Numerical algorithm without saturation for solving problems of mathematical physics and mechanics were constructed and investigated in [27-38]. This paper develops and generalizes results of the papers [1-6].

\section{Rational spectral problem}

Introduce functions $p(x), r(x), x \in \bar{\Omega}$, and assume that there exist positive numbers $\alpha_{i}, \quad \beta_{i}, \quad i=1,2$, satisfying the following conditions $\alpha_{1} \leq p(x) \leq \alpha_{2}, \quad \beta_{1} \leq r(x) \leq \beta_{2}$, $x \in \bar{\Omega}$. We also define nonnegative numbers $K$ and $M$.

Introduce the following linear parameter eigenvalue problems. 
For $\mu \in(0, \sigma)$, find numbers $\gamma(\mu)$ and nonzero functions $w(x), x \in \bar{\Omega}$, such that

$$
\begin{gathered}
\left(p(x) w^{\prime}(x)\right)^{\prime}+\gamma(\mu) r(x) w(x)=0, x \in \Omega, \\
w(0)=p(l) w^{\prime}(l)-\gamma(\mu)(\sigma-\mu)^{-1} K w(l)=0 .
\end{gathered}
$$

For $\mu \in(\sigma, \infty)$, find numbers $\gamma(\mu)$ and nonzero functions $w(x), x \in \bar{\Omega}$, such that

$$
\begin{aligned}
& \left(p(x) w^{\prime}(x)\right)^{\prime}+\gamma(\mu) r(x) w(x)=0, x \in \Omega, \\
& w(0)=p(l) w^{\prime}(l)-\mu(\sigma-\mu)^{-1} K w(l)=0 .
\end{aligned}
$$

The linear parameter eigenvalue problem (9), (10), has positive simple eigenvalues $\gamma_{m}(\mu), \mu \in(0, \sigma), m=1,2, \ldots$ The linear parameter eigenvalue problem (11), (12), has positive simple eigenvalues $\gamma_{m}(\mu), \mu \in(\sigma, \infty), m=2,3, \ldots$ The limit eigenvalue problem (7), (8), has positive simple eigenvalues $\lambda_{m}^{(0)}, m=1,2, \ldots$ Set $\gamma_{1}(\mu)=0$, $\mu \in[\sigma, \infty)$, and $\gamma_{m}(\sigma)=\lambda_{m-1}^{(0)}, \quad m=2,3, \ldots$ Then the functions $\gamma_{m}(\mu), \mu \in[0, \infty)$, $m=1,2, \ldots$, are continuous and non-increasing. Therefore, the functions $\mu-\gamma_{m}(\mu)$, $\mu \in[0, \infty), \quad m=1,2, \ldots$, are continuous and increasing. Consequently, each of the equations $\mu-\gamma_{m}(\mu)=0, \mu \in[0, \infty), m=1,2, \ldots$, has a unique root $\lambda_{m}, m=1,2, \ldots$ These roots are eigenvalues of the rational spectral problem (5), (6).

Theorem 1. The rational spectral problem (5), (6), has positive simple eigenvalues $\lambda_{m}$, $m=1,2, \ldots$ and corresponding eigenfunctions $u_{m}=u_{m}(x), \int_{0}^{l} r(x) u_{m}(x) u_{m}(x) \mathrm{d} x=1$, $m=1,2, \ldots$

The proof of this theorem uses the statements of problem (7), (8), parameter spectral problems (9), (10), and (11), (12).

\section{Numerical experiments}

Let us define $x_{j}=j h, \quad 0 \leq j \leq N, \quad h=l / N$. Denote $p_{j}=p\left(x_{j}\right), \quad r_{j}=r\left(x_{j}\right)$, $y_{x, j}=\left(y_{j+1}-y_{j}\right) / h, \quad y_{\bar{x}, j}=\left(y_{j}-y_{j-1}\right) / h$. Approximate the differential rational spectral problem (5), (6), by using the finite difference method

$$
\begin{gathered}
-\left(p y_{x}\right)_{\bar{x}, j}-\left(p y_{\bar{x}}\right)_{x, j}=\lambda^{h} 2 r_{j} y_{j}, 1 \leq j \leq N-1, \\
y_{0}=0, \frac{1}{2 h} p_{N-1} y_{x, N-1}+\frac{1}{2 h} p_{N} y_{\bar{x}, N}+\frac{1}{h} \frac{\lambda^{h}}{\lambda^{h}-\sigma} K y_{N}=\lambda^{h} \frac{1}{2} r_{N} y_{N} .
\end{gathered}
$$

Let us denote $[y, z)=\sum_{i=0}^{N-1} h y_{i} z_{i},(y, z]=\sum_{i=1}^{N} h y_{i} z_{i}$. 
Theorem 2. The finite difference rational spectral problem (13), (14), has eigenvalues $\lambda_{1}^{h}<\lambda_{2}^{h}<\ldots<\lambda_{N+1}^{h}$ and corresponding eigenvectors $y^{(m)}=\left(y_{0}^{(m)}, y_{1}^{(m)}, \ldots, y_{N}^{(m)}\right)^{\mathrm{T}}$, $1 \leq m \leq N+1$, satisfying the following conditions

$$
\begin{gathered}
\frac{1}{2}\left[p y_{x}^{(m)}, y_{x}^{(m)}\right)+\frac{1}{2}\left(p y_{\bar{x}}^{(m)}, y_{\bar{x}}^{(m)}\right]+\frac{\lambda_{m}^{h}}{\lambda_{m}^{h}-\sigma} K\left(y_{N}^{(m)}\right)^{2}=\lambda_{m}^{h}, \\
\left(r y^{(m)}, y^{(m)}\right]+\left[r y^{(m)}, y^{(m)}\right)=2,
\end{gathered}
$$

for $1 \leq m \leq N+1$.

Theorem 3. For approximate eigenvalues and corresponding eigenfunctions the estimates $\left|\lambda_{m}^{h}-\lambda_{m}\right| \leq c h^{2}$ and $\left\|y^{(m)}-u^{(m)}\right\|_{h} \leq c h^{2}$ hold, where $c$ is a constant independent $\quad$ of $\quad h, \quad\left\|u^{(m)}-y^{(m)}\right\|_{h}=\max _{j=0,1, \ldots, N}\left|u_{j}^{(m)}-y_{j}^{(m)}\right|$, $\left(r u^{(m)}, y^{(m)}\right]+\left[r u^{(m)}, y^{(m)}\right)>0, u_{j}^{(m)}=u_{m}\left(x_{j}\right), 0 \leq j \leq N, 1 \leq m<N+1$.

Theorems 2 and 3 can be established by applying theoretical results [1-6].

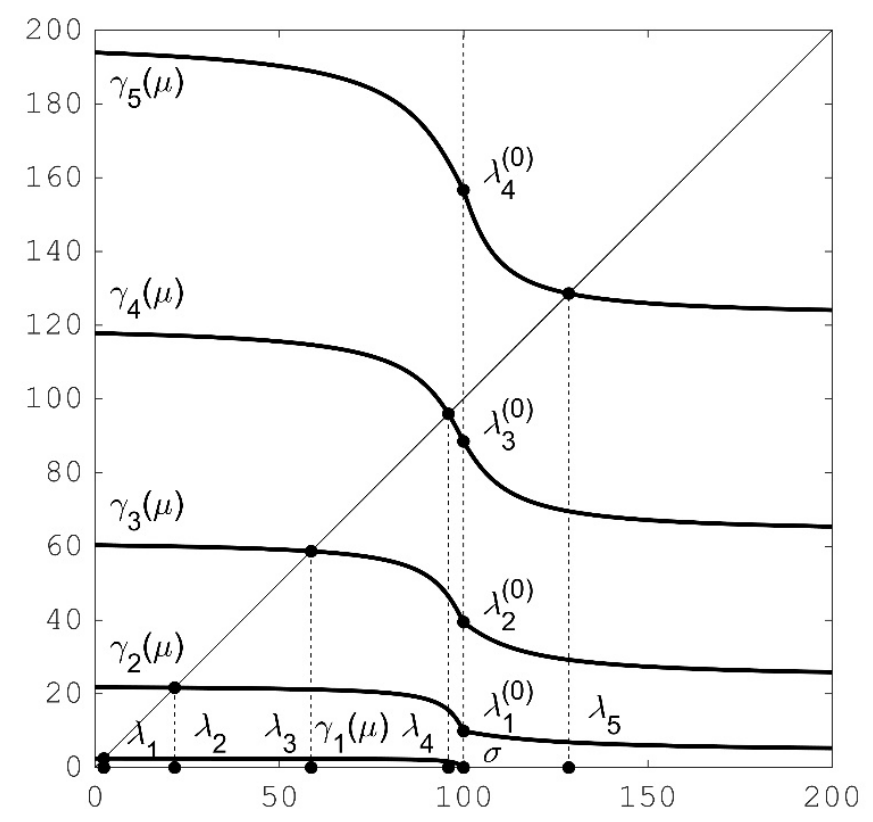

Fig. 1. Eigenvalues of the rational spectral problem.

Figure 1 shows the experimental results for a model problem with constant coefficients. To illustrate Theorems 1, 2 and 3, we solve problem (5), (6), for $l=1, M=0.01, K=1$, $p=1, \quad r=1$. We apply the approximate method (13)-(16) on the uniform mesh for $N=40$. Figure 1 shows the functions $\gamma_{k}(\mu), 1 \leq k \leq 5$, the eigenvalues $\lambda_{k}, 1 \leq k \leq 5$, of problem (5), (6), the eigenvalues $\lambda_{j}^{(0)}, 1 \leq j \leq 4$, of problem (7), (8). We see that the experimental results are consistent with Theorems 1,2 and 3; namely, $\gamma_{1}(\mu) \rightarrow 0$ as 
$\mu \rightarrow \sigma, \gamma_{k+1}(\mu) \rightarrow \lambda_{k}^{(0)}$ as $\mu \rightarrow \sigma, 1 \leq k \leq 4$. Theoretical and experimental results of the paper can be developed for the problems on eigenvibrations of complex mechanical constructions with systems of harmonic oscillators.

This work was supported by Russian Foundation for Basic Research, project nos. 20-31-90086, 2008-01154.

\section{References}

1. Osborn J E 1975 Math. Comp. 29 (131) 712-25

2. $\quad$ Bramble J H and Osborn J E 1973 Math. Comp. 27 (123) 525-49

3. Knyazev A V and Osborn J E 2006 SIAM J. Numer. Anal. 43 (6) 2647-67

4. Sakurai T and Sugiura H 2003 J. Comput. Appl. Math. 159 119-28

5. $\quad$ Karma O 1996 Numer. Funct. Anal. Optim. 17 365-87

6. Karma O 1996 Numer. Funct. Anal. Optim. 17 389-408

7. Knyazev A V and Neymeyr K 2003 Linear Algebra Appl. 358 (1-3) 95-114

8. Knyazev A V and Neymeyr K A 2003 Electr. Trans. Numer. Anal. 15 38-55

9. Neymeyr K A 2001 Linear Algebra Appl. 322 (1-3) 61-85

10. Neymeyr K A 2001 Linear Algebra Appl. 322 (1-3) 87-104

11. Ovtchinnikov E E 2008 J. Comput. Phys. 227 (22) 9477-97

12. Ovtchinnikov E E 2008 SIAM J. Numer. Anal. 46 (5) 2567-92

13. Ovtchinnikov E E 2008 SIAM J. Numer. Anal. 46 (5) 2593-619

14. Ovtchinnikov E E 2006 SIAM J. Numer. Anal. 43 (6) 2668-89

15. Van Beeumen R, Meerbergen K and Michiels W 2013 SIAM J. Sci. Comput. 35 (1) A $327-50$

16. Beyn W-J, Effenberger C and Kressner D 2011 Numer. Math. 119 (3) 489516

17. Huang X, Bai Z and Su Y 2010 J. Comput. Appl Math. 28 (2) 218-34

18. Jarlebring E 2012 Linear Algebra Appl. 436 (10) 3943-53

19. Jarlebring E and Michiels W 2011 BIT 51 (4) 937-57

20. Sleijpen G L, Booten A G, Fokkema D R and van der Vorst H A 1996 BIT 36 (3) 595-633

21. Szyld D B and Xue F 2013 Numer. Math. 123 (2) 333-62

22. Andrew A L, Chu K E and Lancaster P 1995 Computing 55 91-111

23. Arbenz P and Gander W 1986 Computing 36 205-15

24. Asakura J, Sakurai T, Tadano H, Ikegami T and Kimura K 2009 JSIAM Letters $152-5$

25. Jarlebring E, Meerbergen K and Michiels W 2012 Numer. Math. 122 169-95

26. Su Y and Bai Z 2011 SIAM J. Matrix Anal. Appl.32 201-16

27. Algazin S D and Babenko K I 1982 J. Appl. Math. Mech. 46 (6) 814-8

28. Algazin S D 1983 Siber. Math. J. 24 (2) 155-9

29. Algazin S D 1995 Comp. Math. Math. Phys. 35 (3) 321-30

30. Algazin S D 1995 Comp. Math. Math. Phys. 35 (4) 477-82

31. Algazin S D and Kiiko I A 2002 Dokl. Phys. 47 (3) 254-5

32. Algazin S D and Kiiko I A 2002 Dokl. Akad.Nauk. 383 (3) 343-6

33. Algazin S D 2008 Dokl. Math. 78 (2) 675-8 
34. Algazin S D 2013 Math. Models Comp. Simulat. 5 (6) 520-6

35. Algazin S D 2013 J. Appl. Mech. Tech. Phys. 54 (2) 287-94

36. Algazin S D 2019 Dokl. Math. 99 (3) 260-2

37. Algazin S D 2006 J. Appl. Mech. Tech. Phys. 47 (3) 433-8

38. Algazin S D 2006 J. Appl. Mech. Tech. Phys. 47 (4) 573-81 